\title{
Moving Target Detection and Characterization with circular SAR
}

\author{
Leonid Perlovsky, Roman Ilin, Ross Deming*, Rob Linnehan, Freeman Lin \\ Sensors Directorate \\ Air Force Research Laboratory \\ Hanscom AFB, MA \\ Leonid.Perlovsky@hanscom.af.mil \\ On-site Research Contractor: Solid State Scientific Corp.
}

\begin{abstract}
In this work, we examine ground target detection and characterization from radar data, which incorporates a modelbased optimization method called dynamic logic (DL). We apply our methodology to a prototype airborne radar platform called Gotcha, developed by the Air Force Research Laboratory/Sensors Directorate/Automatic Target Recognition Division in recent years. The aircraft traces out a circular path around an area of interest, and the onboard, side-looking radar transmits and receives energy at a constant pulse repetition frequency, while the main beam direction is maintained at a fixed aim point on the ground. Data collected during any appropriate length arc of the flight path can be used to create synthetic aperture radar (SAR) images of the ground. The data can also be used for ground moving target indication (GMTI) and provide Doppler/Range imagery of the same ground area. Our approach combines the computation of Range-Doppler surfaces and a variable target velocity backprojection SAR method. Potential targets are detected using multiple backprojection images and features are extracted using adaptive mixture models. We demonstrate the feasibility of our approach using target truth information provided with the Gotcha dataset. We outline the steps toward implementing a comprehensive automatic target tracking solution based on presented methodology.
\end{abstract}

\section{INTRODUCTION}

The ability to conduct efficient surveillance and tracking of ground vehicles and dismounts is essential for modern, asymmetric warfare. This need is emphasized by a number of ongoing research programs devoted to developing systems that exploit RF signatures of ground vehicles and dismounts. Efficient and intelligent automatic data processing algorithms are required for real-time data analysis, target

characterization and detection of suspicious events, such as activities related to IED emplacement.

Although relevant and valuable information may be collected by sensors, subtle variations and extremely large amounts of data make it challenging to exploit that information in an efficient and effective manner. The exploitation challenge is compounded by the complex operational environments where modern warfare occurs, such as crowded urban areas or mountainous terrain.

To address this urgent need, we are developing radar exploitation tools using Dynamic Logic (DL), a general framework for model-based signal processing and optimization [1]. More specifically, we are tailoring DL to the problem of simultaneous detection, classification, and tracking of ground targets with respect to radar data collected from a circular airborne path. Previously, we used DL to develop algorithms for detection and tracking targets in conventional GMTI radar data having low signal-tointerference ratios with significant improvement [2-6].

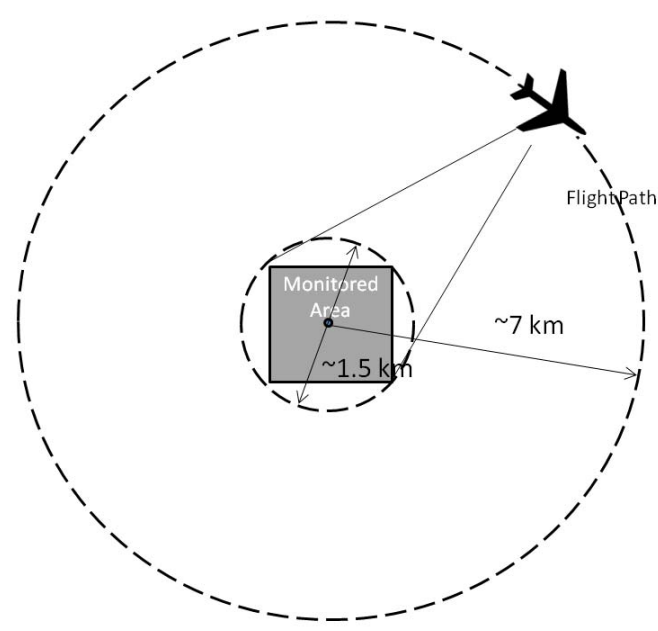

Figure 1. Schematic illustration of the circular radar data collection.

In the following sections we present our general approach to target characterization and describe exploitation using the Gotcha radar experimental data, which was collected during a series of tests conducted by AFRL at Wright-Patterson Air Force base [7][8]. We then show an example of target detection using DL and verify performance with quantitative 
truth data. Finally, we discuss the results of our experiment and outline the directions of future research in Gotcha radar characterization we intend to pursue.

\section{RADAR DATA PROCESSING}

The flight path of the data collection aircraft is illustrated in Fig. 1. Side looking radar emits a beam illuminating an area on the ground with a diameter close to $1.5 \mathrm{~km}$, where the slant range to the center of this circle is about $10 \mathrm{~km}$. The radar operates in spotlight mode so the same aim point of the antenna is maintained during the full circular orbit. The radar characteristics are selected to achieve a region of unambiguous range resolution close to $1.5 \mathrm{~km}$ long. The radar uses stretch mode of operation over a range of frequencies at $\mathrm{X}$-band. Each returned pulse is stored in a series of I and Q components, which constitute the raw data available for exploitation.

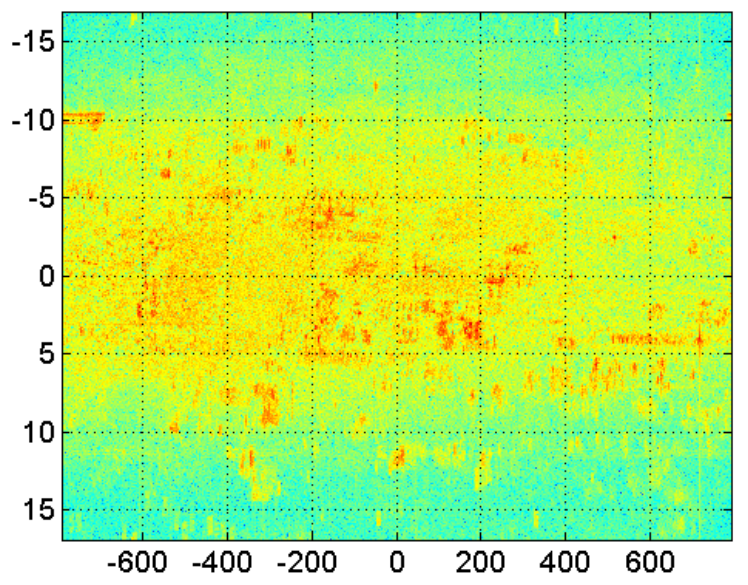

Figure 2. Monitored area in Range/Doppler space. Horizontal axes is Range in meters, vertical axes is Doppler in $\mathrm{m} / \mathrm{sec}$.

SAR achieves high resolution by processing multiple echo waveforms received at different antenna positions. In our case the consecutive antenna positions are located on an arc of the aircraft path. We select a coherent processing interval (CPI) containing $\mathrm{N}_{\mathrm{t}}$ pulses, each pulse containing $\mathrm{N}_{\mathrm{f}}$ frequencies. Thus the CPI data is an $\mathrm{N}_{\mathrm{t}}$ by $\mathrm{N}_{\mathrm{f}}$ array of complex I $+\mathrm{Qi}$ values. Standard Range-Doppler processing consists of performing 2-dimensional Discrete Fourier Transform (DFT) of the data for each CPI and appropriate scaling of the units[10]. An example of such Range/Doppler image from the Gotcha data collection in 2006 is shown in Fig. 2. The range axis is scaled in meters with the origin at the antenna aimpoint. The Doppler axis is scaled in meters per second and also has its origin at the aimpoint since the radial velocity of the aimpoint relative to the aircraft is zero. All other points on the ground have induced radial velocity which depends on their location relative to the aimpoint. This induced velocity explains a large central region of the Range/Doppler image. The energy of the beam is focused on the aimpoint and therefore weakens toward the edges of the image showing decreased brightness of the images of static structures (buildings, roads, etc). Targets possessing radial velocity relative to the aircraft and moving relatively fast (at least 10 $\mathrm{m} / \mathrm{sec}$ in the radial direction) can be detected since they appear outside the central clutter region. However,the slowly moving targets are located within the central region. They can be detected by considering a series of Range/Doppler images and identifying bright patches moving relative to the static structures. Such a task is complicated by the presence of large number of stationary objects in each image and the jitter induced by the aircraft motion. Dynamic Logic approach described below provides a good solution to this problem.

As mentioned above, jitter from air turbulance onboard the aircraft causes the antenna aimpoint to randomly fluctuate around the desired location, which in turn makes corellations between processed imagery and ground truth data more difficult. A backprojection algorithm [9] allows the formation of more precise images due to specifics of Gotcha data collection. This algorithm uses only the range information in the stored data. The post-processing done on board of the data collection aircraft includes motion compensation adjusting the phase of each pulse based on the actual range to the aimpoint. Thus the range information contained in the data is exact. This fact is exploited by the backprojection algorithm to produce high resolution images of the static structures on the ground. Just like the Range/Doppler imagery, the positions of the moving targets are shifted due to additional Doppler component. For example a moving vehicle will appear moving in the areas without roadways. If the radial velocity of the vehicle is known the backprojection algorithm can be modified to adjust the vehicle position. The unknown velocity can be estimated using the Range/Doppler images described above and used to produce motion compensated backprojection images taking advantage of the precise knowledge of the range. The detection of the moving target still requires a good data processing approach to separate targets from static clutter, provided by Dynamic Logic algorithm in our case.

As an illustration, consider a backprojection image in Fig. 3. This image is obtained by processing $\mathrm{Nt}=2000$ pulses from Gotcha data set [7]. A car stopped at the intersection appears at its true geographical position. Fig. 4 shows a patch of the roadway to the left of the intersection with the same car approaching the intersection with approximately constant speed. The car cannot be seen on the image. We use the GPS information to estimate the velocity of the car to adjust the position of the car. Figure 5 shows a sequence of 4 images obtained by adjusting the backprojection algorithm to the car's known velocity. The sequence spans approximately $1 \mathrm{sec}$ and contains the motion of the car between the first and the second red dot in Fig. 4 moving from left to right. The level of noise in this image sequence underlines the need for a robust automatic detection method. 


\section{DYNAMIC LOGIC}

Dynamic Logic [1] provides an elegant framework for simultaneously detecting, tracking, and classifying multiple targets using an adaptive mixture of "competing" model components.

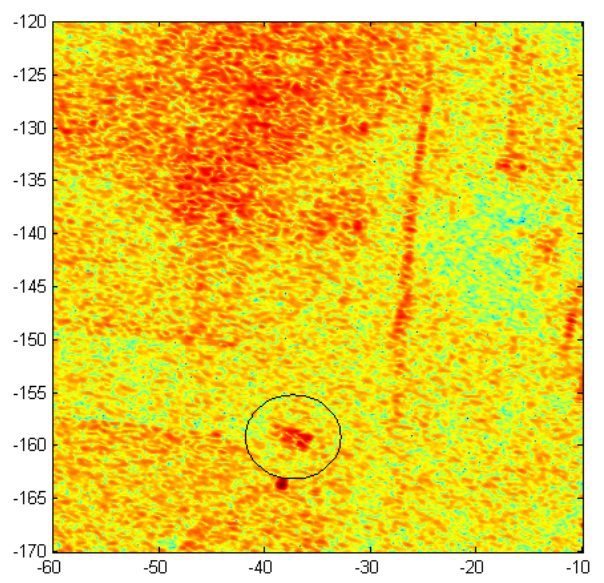

Figure 3. Image of a car stopped at an intersection obtained using backprojection algorithm. The position of the car is verified by GPS data. The axes are in meters relative to the antenna aimpoint.

Model parameters converge to the solution which maximizes the similarity between the models and the data, providing track estimates and features for target classification.

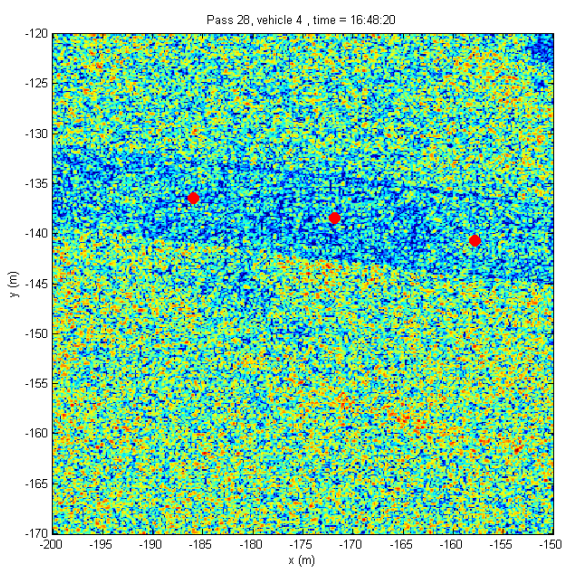

Figure 4. Backprojection image of the same roadway as appears in Fig. 3 approaching the intersection from the left. The car is known to move towards the intersection with ground velocity $\sim 14 \mathrm{~m} / \mathrm{sec}$. The red dots show the GPS positions of the car with $1 \mathrm{sec}$ increment. The car itself does not appear on this image.
The main components of the DL framework are the input data and the parametric models.
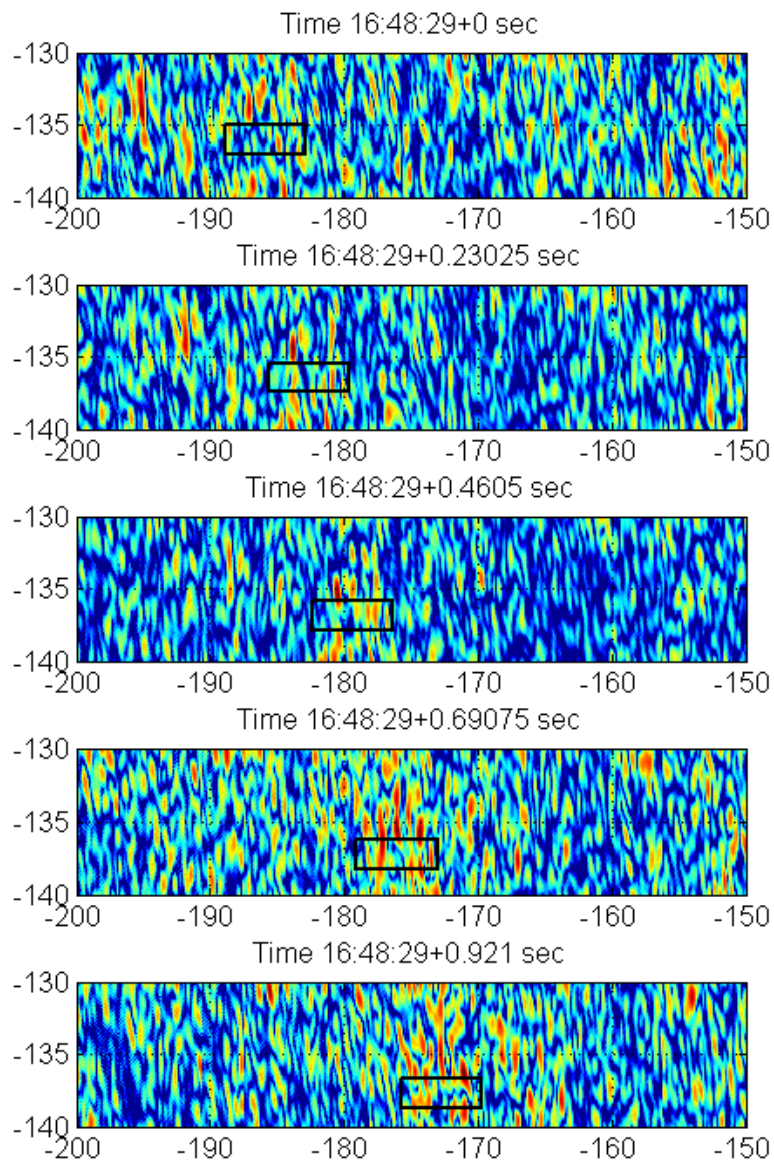

Figure 5. Sequence of backprojection images of the road in Fig. 4 adjusted by the radial velocity of the vehicle. The road itself does not appear in the image. The rectangle shows the true position of the vehicle.

We denote the input pixel by $\mathbf{x}_{n}, n=1$..N. Each pixel is a vector with components giving its image coordinates, color, and timestamp. There are a total of $\mathrm{N}$ data points coming from multiple frames. We denote the models by $\mathrm{M}_{\mathrm{h}}, \mathrm{h}=1 . . \mathrm{H}$, where $\mathrm{H}$ is the total number of models. Each model $\mathrm{h}$ depends on parameters $\mathbf{S}_{\mathrm{h}}: \mathrm{M}_{\mathrm{h}}=\mathrm{M}_{\mathrm{h}}\left(\mathbf{S}_{\mathrm{h}}\right)$. Models $\mathrm{M}_{\mathrm{h}}$ are probabilistic, with conditional probability density function (PDF) of the data element $\mathbf{x}_{n}$ given the model $M_{h}$ denoted by $\mathrm{l}(\mathrm{n} \mid \mathrm{h})$. Note that this is a function of model parameters. The total conditional likelihood of the data $\mathrm{x}=\left\{\mathbf{x}_{\mathrm{n}}\right\}$ given all the models $M=\left\{M_{h}\right\}$ is expressed as follows. 
Input Images
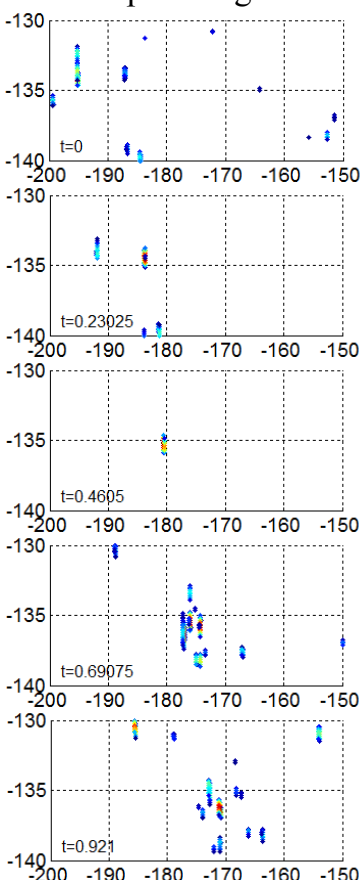

Iteration 2
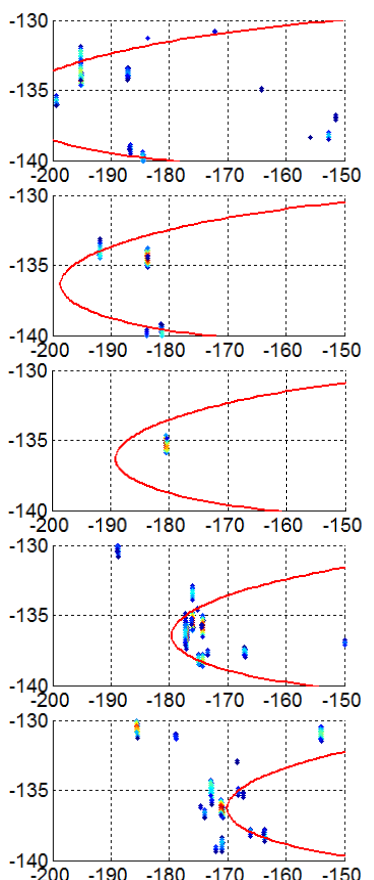

Iteration 5
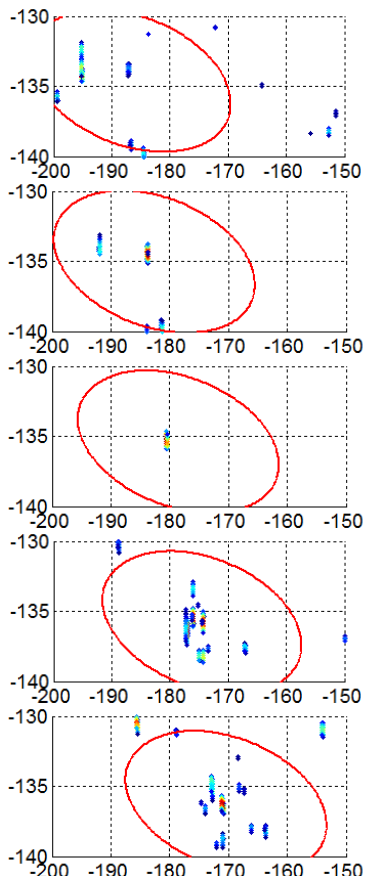

Iteration 10
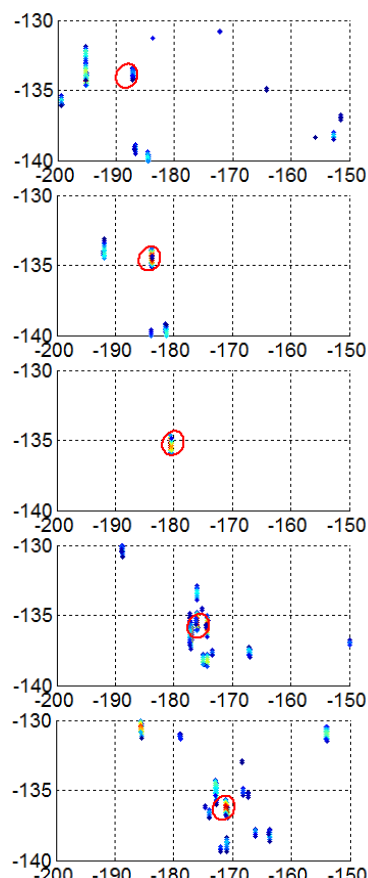

Figure 6. Iterations of the DL algorithm; first column contains 5 input frames. These images are the same as in Fig. 5 after the application of threshold to remove most of the noise. The images are analyses by the DL algorithm to detect a target track. Columns 2, 3, and 4 illustrate the convergence of the algorithm. The red ellipse is the track model. The clutter model is not shown. The algorithm converges to the target track corresponding to GPS truth after 10 iterations .

$$
L(x \mid M)=\prod_{n=1}^{N}\left[\sum_{h=1}^{H} r_{h} l(n \mid h)\right]
$$

Here $\mathrm{r}_{\mathrm{h}}$ is the prior probability of encountering a model of class $\mathrm{h}$. Maximization of (1) with respect to model parameters results in the maximum likelihood parameter estimate. This is achieved by using the following iterative procedure. First, the parameters are set to some initial values. Second, the following association weights are computed using the parameter values

$$
f(n \mid h)=\frac{r_{h} l(n \mid h)}{\sum_{h^{\prime}=1}^{H} r_{h^{\prime}} l\left(n \mid h^{\prime}\right)}
$$

Next the new parameter values are determined by solving the following equation.

$$
\sum_{n=1}^{N} f(h \mid n) \frac{\partial \log l(n \mid h)}{\partial S_{h}}=0
$$

The algorithm iterates between (2) and (3) until estimated parameters stop changing or some other stopping criteria are satisfied. Practical application of this algorithm consists of specifying the exact form of PDF (1) for each model type. The track model PDF consists of three components: object model, feature model and motion model. This is expressed as the following product of PDF's.

$$
l(n \mid h)=l_{\text {obj }}(n \mid h) l_{\text {feat }}(n \mid h) l_{\text {mot }}(n \mid h)
$$

Specific form of PDF depends on application. The main advantage of using this algorithm is that it is more efficient due to its ability to avoid combinatorial complexity. This is achieved by employing probabilistic association weights (2) and enforcing gradual and simultaneous convergence of association probabilities and model parameters to their optimal estimates.

In this work we use the algorithm to detect moving targets in sequences of images. Targets can be described as blobs moving across the image with constant velocity. The images contain clutter in the form of other blobs and individual points randomly appearing is different locations. The brightness of the clutter varies with some blobs appearing brighter than the targets. 
We use linear motion model, Gaussian feature model and Gaussian object model given by the following equations.

$$
\left.\begin{array}{c}
l_{\text {obj }}=\frac{1}{\sqrt{(2 \pi)^{2}\left|C O_{h}\right|}} e^{-0.5\left(x_{n}-x c_{h n}\right)^{T}\left(C O_{h}\right)^{-1}\left(x_{n}-x c_{h n}\right)} \\
l_{\text {feat }}=\frac{1}{\sqrt{(2 \pi)^{d}\left|C Y_{h}\right|}} e^{-0.5\left(y_{n}-Y_{h}\right)^{T}\left(C Y_{h}\right)^{-1}\left(y_{n}-Y_{h}\right)} \\
l_{\text {mot }}=\frac{1}{\sqrt{(2 \pi)^{2}\left|C X_{h}\right|}} e^{-0.5\left(x c_{h n}-X_{h n}\right)^{T}\left(C X_{h}\right)^{-1}\left(x c_{h n}-X_{h n}\right)}
\end{array}\right\}
$$

The motion is incorporated in the motion PDF using the following equation.

$$
X_{h n}=X 0_{h}+V_{h} t_{n}
$$

Here the model parameters are the mean and covariance of each of the models and the initial position and velocity of the track. The target PDF is therefore obtained by substituting (5) into (4). The clutter model uses Gaussian feature model. There are no object and motion models for clutter, so we model the position of the clutter points with uniform density. Thus the clutter model PDF is given as follows.

$$
l(n \mid c)=\frac{1}{A} \frac{1}{\sqrt{(2 \pi)^{d}\left|C Y_{c}\right|}} e^{-0.5\left(y_{n}-Y_{c}\right)^{T}\left(C Y_{c}\right)^{-1}\left(y_{n}-Y_{c}\right)}
$$

Here $\mathrm{A}$ is the area of the image. The dimensionality of the feature $d=1$ in our case and we use model index $\mathrm{c}$ instead of $\mathrm{h}$ to emphasize that this is the clutter model PDF.

We applied the algorithm to the sequence of images in Fig .6. The images were thresholded to get rid of most but not all background noise. Thresholding is not necessary for application of the algorithm and was done to speed up the processing. The algorithm quickly converged to the target track separating image points coming from the target from clutter points. The run time of this example, implemented in Matlab, is 2.7 seconds on a Dell desktop computer with $3 \mathrm{GHz}$ processor and $4 \mathrm{~GB}$ of RAM. This can be improved by several orders of magnitude with more efficient implementation and specialized hardware.

\section{DISCUSSION}

The goal of this work was to describe our current approach to the Gotcha data exploitation. We discussed two ways of forming SAR imagery from the data. We gave a brief overview and an example of usage of the Dynamic Logic algorithm for detection and feature extraction from the SAR imagery. The long term purpose of this research is the development of comprehensive solution for detection and tracking of moving targets - vehicles and dismounts. We propose to combine the Range/Doppler and the backprojection SAR imagery to exploit the advantages of both methods to achieve robust tracking. The combined algorithm will operate as follows.

1) The ground radial speed of the moving target is estimated using DL algorithm applied to Range/Doppler images. The Range/Doppler images can be computed relatively fast.

2) The estimated target speed is plugged into the backprojection algorithm to obtain the SAR images of the moving target. The precision of backprojection images results in exact positioning of the target.

3) Use DL algorithm to detect and estimate the parameters of the track and other features of the target such as its radar cross section.

4) Once the target is detected the subsequent tracking can be done by extending the track over the future backprojection images and using DL and parameters estimated during detection.

\section{REFERENCES}

[1] Perlovsky, L.I, "Neural Networks and Intellect: using model based concepts", 2001, New York: Oxford University Press.

[2] Perlovsky L.I. and Deming R.W, "Neural networks for improved tracking", Neural Networks IEEE Transactions on, Vol 18, Issue 6, Nov. 2007 Page(s):1854 - 1857

[3] Deming, R.W. and Perlovsky, L.I, “GMTI tracking improvement by $20 \mathrm{~dB}, 52 \mathrm{nd}$ Annual Meeting of the MSS Tri-Service Radar Symposium, 19-23 June, 2006, Lincoln Laboratory, Lexington, MA, 2006.

[4] Deming, R.; Schindler, J.; Perlovsky, L., "MultiTarget/Multi-Sensor Tracking using Only Range and Doppler Measurements," Aerospace and Electronic Systems, IEEE Transactions on, vol.45, no.2, pp.593611, April 2009

[5] Kozma, R.; Perlovsky, L.; Ankishetty, J.S., "Detection of propagating phase gradients in EEG signals using Model Field Theory of non-Gaussian mixtures," Neural Networks, 2008. IJCNN 2008. (IEEE World Congress on Computational Intelligence). IEEE International Joint Conference on, vol., no., pp.3524-3529, 1-8 June 2008

[6] Deming R, Perlovsky L.I., Linnehan R, "Concurrent Classification and Tracking Dismounts using Adaptive Mixture Models," DARPA RF Dismount Characterization Workshop, Arlington, VA, Dec. 5-7, 2007

[7] Curtis H. Casteel, Jr., LeRoy A. Gorham, Michael J. Minardi, Steven M. Scarborough, Kiranmai D. Naidu, and Uttam K. Majumder, "A challenge problem for 2D/3D imaging of targets from a volumetric data set in an urban environment", Proc. of SPIE 6568, 65680D (2007)

[8] Majumder, U.; Soumekh, M.; Minardi, M.; Scarborough, S.; Gorham, L.; Casteel, C.; Judge, M.; Kirk, J., "Synthetic aperture radar moving target indication 
processing of along-track monopulse nonlinear gotcha data" Radar Conference, 2009 IEEE, 4-8 May 2009

Page(s):1-6

[9] LeRoy A. Gorham, Uttam K. Majumder, Peter Buxa, Mark J. Backues, and Andrew C. Lindgren,

"Implementation and analysis of a fast backprojection algorithm", Proc. SPIE 6237, 62370G (2006),

DOI:10.1117/12.674024

[10] Richards, M.A., "Fundamentals of radar signal

processing”, McGraw-Hill, 2005, Ch. 5, p. 253 